\title{
PERMUTATION POLYNOMIALS OF DEGREE 8 OVER FINITE FIELDS OF CHARACTERISTIC 2
}

\author{
XIANG FAN
}

\begin{abstract}
Up to linear transformations, we obtain a classification of permutation polynomials (PPs) of degree 8 over $\mathbb{F}_{2^{r}}$ with $r>3$. By [J. Number Theory 176 (2017) 46-66], a polynomial $f$ of degree 8 over $\mathbb{F}_{2}{ }^{r}$ is exceptional if and only if $f-f(0)$ is a linearized PP. So it suffices to search for non-exceptional PPs of degree 8 over $\mathbb{F}_{2^{r}}$, which exist only when $r \leqslant 9$ by a previous result. This can be exhausted by the SageMath software running on a personal computer. To facilitate the computation, some requirements after linear transformations and explicit equations by Hermite's criterion are provided for the polynomial coefficients. The main result is that a non-exceptional PP $f$ of degree 8 over $\mathbb{F}_{2^{r}}$ (with $r>3$ ) exists if and only if $r \in\{4,5,6\}$, and such $f$ is explicitly listed up to linear transformations.
\end{abstract}

\section{INTRODUCTION}

Let $\mathbb{F}_{q}$ denote the finite field of characteristic $p$ and order $q=p^{r}$, and let $\mathbb{F}_{q}^{*}=\mathbb{F}_{q} \backslash\{0\}$. Reserve the letter $x$ for the indeterminate of the polynomial ring $\mathbb{F}_{q}[x]$ with coefficients in $\mathbb{F}_{q}$. We call $f \in \mathbb{F}_{q}[x]$ a permutation polynomial $(\mathrm{PP})$ over $\mathbb{F}_{q}$ if the induced map $a \mapsto f(a)$ permutes $\mathbb{F}_{q}$. Initiated by Hermite [9] and Dickson [4] in the 19th century, the study of PPs has drawn much attention, with more and more classes of PPs (with either nice appearance or certain desired properties) found or constructed. Some classes of them have significant applications in wide areas of mathematics and engineering such as cryptography, coding theory, combinatorial designs. However, the non-trivial problems of classification of PPs (of certain prescribed forms) are still challenging.

Especially, the classification of PPs of a given degree $d$ over an arbitrary $\mathbb{F}_{q}$ is complete known only for $d \leqslant 7$ :

- by Dickson's 1896 thesis [4] for $d \leqslant 5$ with any $q$, and for $d=6$ with any odd $q$;

- by Li, Chandler and Xiang [10] in 2010 for $d=6$ or 7 with $q=2^{r}$ (for any $r \geqslant 3$ );

- by the author's recent work [5] for $d=7$ with any odd prime power $q$.

The present paper aims for a classification of PPs of degree 8 over $\mathbb{F}_{2^{r}}$ (with any integer $r>3$ ) up to linear transformations.

Similar to [5], our approach here is based on some known results on exceptional polynomials. In this paper, an exceptional polynomial over $\mathbb{F}_{q}$ is defined as a PP over $\mathbb{F}_{q}$ which is also a PP over $\mathbb{F}_{q^{m}}$ for infinitely many integers $m \geqslant 1$. Recall that $\mathbb{F}_{q}$ is of characteristic $p$. A polynomial $\varphi$ in $\mathbb{F}_{q}[x]$ of the form $\varphi(x)=\sum_{s=0}^{t} c_{s} x^{p^{s}}$ (with all $c_{s} \in \mathbb{F}_{q}$ ) is called a linearized polynomial. Clearly, a linearized polynomial $\varphi$ induces a $\mathbb{F}_{p}$-linear map from $\mathbb{F}_{q}$ to itself, so $\varphi$ is a PP over $\mathbb{F}_{q}$ if and only if $\mathbb{F}_{q}^{*}$ contains no root of $\varphi$. On the one hand, a linearized PP $\varphi$ over $\mathbb{F}_{q}$ is always exceptional. Indeed, let $\mathbb{F}_{q^{k}}$ be the splitting field of $\varphi$ over $\mathbb{F}_{q}$, then every $\mathbb{F}_{q^{m}}^{*}$ with $\operatorname{gcd}(m, k)=1$

2000 Mathematics Subject Classification. 11T06, 12 Y05.

Key words and phrases. Permutation polynomial; Exceptional polynomial; Hermite's criterion; SageMath. 
contains no root of $\varphi$. On the other hand, the recent work of Bartoli, Giulietti, Quoos and Zini [1, Proposition 7.1] showed that a polynomial $f \in \mathbb{F}_{2^{r}}[x]$ (with $r>3$ ) of degree 8 is exceptional if and only if $f(x)-f(0)$ is linearized and has no root in $\mathbb{F}_{2^{r}}^{*}$. Also note an explicit criterion $[4, \S \S 58]$ for a linearized polynomial over $\mathbb{F}_{q}$ to have no root in $\mathbb{F}_{q}^{*}$. Combining them, we get the following explicit determination of exceptional polynomials of degree 8 over $\mathbb{F}_{2^{r}}$ (with $r>3$ ).

Lemma 1 ([1, Proposition 7.1] and [4, §§58]). A polynomial $f(x)=\sum_{i=0}^{8} a_{i} x^{i} \in \mathbb{F}_{2^{r}}[x]$ (with $r>3$, all $a_{i} \in \mathbb{F}_{2^{r}}$ and $a_{8} \neq 0$ ) is exceptional over $\mathbb{F}_{2^{r}}$ if and only if $a_{7}=a_{6}=a_{5}=a_{3}=0$ (i.e. $f(x)-a_{0}$ is linearized) and $\operatorname{det}\left(c_{i-j}^{p^{j}}\right)_{0 \leqslant i, j \leqslant r-1} \neq 0$, where

$$
\left(c_{0}, c_{1}, c_{2}, c_{3}\right)=\left(c_{-r}, c_{-r+1}, c_{-r+2}, c_{-r+3}\right)=\left(a_{1}, a_{2}, a_{4}, a_{8}\right)
$$

and all other $c_{s}=0$.

Thereafter, to complete the classification of PPs of degree 8 over $\mathbb{F}_{2^{r}}$ (with $r>3$ ), it suffices to search for the non-exceptional ones. It is well-known that a non-exceptional PP of degree $n$ exists over $\mathbb{F}_{q}$ only if $q \leqslant C_{n}$, where $C_{n}$ stands for a constant depending only on $n$. The proof of this fact can be found in $[3,8,14]$ for abstract $C_{n}$, in [7] for $C_{n}=n^{4}$, in [2] for a bound less than $n^{2}(n-2)^{2}$, and in [6] for

$$
C_{n}=\left\lfloor\left(\frac{(n-2)(n-3)+\sqrt{(n-2)^{2}(n-3)^{2}+8 n-12}}{2}\right)^{2}\right\rfloor,
$$

where $\lfloor t\rfloor$ denotes the greatest integer not exceeding a real number $t$. When $n=8$, the last bound is 925 , which indicates the following lemma.

Lemma 2. A non-exceptional PP of degree 8 exists over $\mathbb{F}_{2^{r}}$ only if $r \leqslant 9$.

In this paper, we search for non-exceptional PPs of degree 8 over $\mathbb{F}_{2^{r}}$ (with $4 \leqslant r \leqslant 9$ ), with the help of SageMath [13], an open-source computer algebra system with features covering many aspects of mathematics, including algebra, calculus, combinatorics, graph theory, number theory, numerical analysis and statistics. SageMath uses a syntax resembling Python's, which is easy to understand for readers without prior programming experience. We run all algorithms in this paper on the version 8.6 of SageMath.

Most of our efforts are devoted to reduce the number of searching candidates for non-exceptional PPs. The structure of this paper is as follows. Section 2 investigates linear transformations of polynomials of degree 8 over $\mathbb{F}_{2^{r}}$, and imposes some constraints on the polynomial coefficients after linear transformations. Section 3 establishes Algorithm 1 for explicit equations on coefficients of PPs over $\mathbb{F}_{2^{r}}$, by Hermite's criterion and a multinomial analogue of the Lucas theorem. Combining constraints from Section 2 and equations as outputs of Algorithm 1, we analysis the polynomial coefficients of non-exceptional PPs of degree 8 in Section 4, on a case-by-case basis for $4 \leqslant r \leqslant 9$. It is verified that all PPs of degree 8 over $\mathbb{F}_{2^{r}}$ with $r \in\{7,8,9\}$ are exceptional. For $r \in\{4,5,6\}$, we write explicit SageMath codes to test all remaining candidates for nonexceptional PPs of degree 8 over $\mathbb{F}_{2^{r}}$. We also rewrite the SageMath outputs as Theorem 8, 9, and 10, listing all non-exceptional PPs of degree 8 over $\mathbb{F}_{2^{r}}$, up to linear transformations.

\section{LineAR TRANSFORMATIONS}

To reduce the number of candidates in our search for non-exceptional PPs of degree 8 over $\mathbb{F}_{2^{r}}$, we consider the classification up to linear transformations. We say that two polynomials $f$ and $g$ in $\mathbb{F}_{q}[x]$ are related by linear transformations (linearly related for short) if there exist 
$s, t \in \mathbb{F}_{q}^{*}$ and $u, v \in \mathbb{F}_{q}$ such that $g(x)=s f(t x+u)+v$. Linearly related $f$ and $g$ hold the same degree, and $f$ is a (non-exceptional) PP over $\mathbb{F}_{q}$ if and only if so is $g$.

Each PP of degree 8 over $\mathbb{F}_{2^{r}}$ is linearly ralated to some $f \in \mathbb{F}_{2^{r}}[x]$ in normalized form, i.e. $f(x)=x^{8}+\sum_{i=1}^{7} a_{i} x^{i}$ with all $a_{i} \in \mathbb{F}_{2^{r}}$. As we mentioned before, a linearized PP must be exceptional. So $\left(a_{7}, a_{6}, a_{5}, a_{3}\right) \neq(0,0,0,0)$ for $f$ to be non-exceptional. Furthermore, a case-bycase analysis in Section 4 by Hermite's criterion will show that $\left(a_{7}, a_{6}, a_{5}\right) \neq(0,0,0)$ if $f$ is a non-exceptional PP over $\mathbb{F}_{2^{r}}$ with $4 \leqslant r \leqslant 9$. For later use, up to linear transformations, more constraints on the coefficients $a_{i}$ 's can be imposed by the following Proposition 3 .

Proposition 3. Let e be a generator of the multiplicative group $\mathbb{F}_{2^{r}}^{*}$. For each $a \in \mathbb{F}_{2^{r}}^{*}$, fix an element $\omega(a)$ in the set $\mathbb{F}_{2^{r}} \backslash\left\{u^{2}+a u: u \in \mathbb{F}_{2^{r}}\right\}$. Then each polynomial of degree 8 over $\mathbb{F}_{2^{r}}$ is linearly ralated to some $f(x)=x^{8}+\sum_{i=1}^{7} a_{i} x^{i} \in \mathbb{F}_{2^{r}}[x]$ with all $a_{i} \in \mathbb{F}_{2^{r}}$ satisfying the following requirements $(\mathbf{R} 1) \sim(\mathbf{R} 3)$ :

(R1) $\left(a_{7}, a_{6}\right) \in\{(1,0),(0,1),(0,0)\}$.

(R2) if $\left(a_{7}, a_{6}\right)=(0,1)$, then $a_{4} \in \begin{cases}\{0\} & \text { if } a_{5}=0, \\ \left\{0, \omega\left(a_{5}\right)\right\} & \text { if } a_{5} \neq 0 .\end{cases}$

(R3) if $a_{7}=a_{6}=0 \neq a_{5}$, then $a_{4}=0$ and $a_{5} \in \begin{cases}\{1\} & \text { if } r \text { is odd, } \\ \left\{1, e, e^{2}\right\} & \text { if } r \text { is even. }\end{cases}$

Moreover, suppose $\left(a_{7}, a_{6}, a_{5}\right) \neq(0,0,0)$ and let $g(x)=x^{8}+\sum_{i=1}^{7} a_{i}^{\prime} x^{i} \in \mathbb{F}_{2^{r}}[x]$ with all $a_{i}^{\prime} \in \mathbb{F}_{2^{r}}$ satisfying the same requirements $(\mathbf{R 1}) \sim(\mathbf{R 3})$, then $f$ and $g$ are linearly related if and only if one of the following happens:

(i) $g=f$.

(ii) $g(x)=f\left(x+a_{5}\right)-f\left(a_{5}\right)$ with $\left(a_{7}, a_{6}\right)=(0,1)$ and $a_{5}\left(a_{3}+a_{5}^{3}\right) \neq 0$. In this case,

$$
\left(a_{7}^{\prime}, a_{6}^{\prime}, a_{5}^{\prime}, a_{4}^{\prime}, a_{3}^{\prime}, a_{2}^{\prime}, a_{1}^{\prime}\right)=\left(a_{7}, a_{6}, a_{5}, a_{4}, a_{3}, a_{2}+a_{3} a_{5}+a_{5}^{4}, a_{1}+a_{3} a_{5}^{2}+a_{5}^{5}\right) .
$$

(iii) $r$ is even, $a_{7}=a_{6}=a_{4}=0 \neq a_{5}$ and $g(x)=t^{-8} f(t x)$ with $t \in\left\{e^{\frac{2^{r}-1}{3}}, e^{\frac{2\left(2^{r}-1\right)}{3}}\right\}$.

Proof. By definition, each polynomial of degree 8 over $\mathbb{F}_{2^{r}}$ is linearly ralated to some $h(x)=$ $x^{8}+\sum_{i=1}^{7} c_{i} x^{i} \in \mathbb{F}_{2^{r}}[x]$ with all $c_{i} \in \mathbb{F}_{2^{r}}$. If $c_{7} \neq 0$, let $f(x)=c_{7}^{-8} h\left(c_{7} x+c_{7}^{-1} c_{6}\right)-c_{7}^{-8} h\left(c_{7}^{-1} c_{6}\right)=$ $x^{8}+x^{7}+\sum_{i=1}^{5} a_{i} x^{i}$. If $c_{7}=0 \neq c_{6}$, let $f(x)=c_{6}^{-4} h\left(c_{6}^{r^{r-1}} x\right)=x^{8}+x^{6}+\sum_{i=1}^{5} a_{i} x^{i}$. If $c_{7}=c_{6}=0$, let $f=h$. In any case, $h$ is linearly related to $f(x)=x^{8}+\sum_{i=1}^{7} a_{i} x^{i}$ with all $a_{i} \in \mathbb{F}_{2^{r}}$ satisfying (R1). Henceforth, we only need to adjust $f$ up to linear transformations, to meet (R2) and (R3).

(R2): Suppose $\left(a_{7}, a_{6}\right)=(0,1)$. Then $f(x)=x^{8}+x^{6}+\sum_{i=1}^{5} a_{i} x^{i}$ can be replaced by

$$
f(x+u)-f(u)=x^{8}+x^{6}+a_{5} x^{5}+\left(u^{2}+a_{5} u+a_{4}\right) x^{4}+\text { lower terms, }
$$

with an arbitrary $u \in \mathbb{F}_{2^{r}}$. If $a_{5}=0$, take $u=a_{4}^{2^{r-1}}$, then $f(x+u)-f(u)$ satisfies (R2). Hereafter assume $a_{5} \neq 0$. Note that $u \mapsto u^{2}+a_{5} u$ gives a $\mathbb{F}_{2^{-}}$-linear map from $\mathbb{F}_{2^{r}}$ to itself, so $\left\{u^{2}+a_{5} u: u \in \mathbb{F}_{2^{r}}\right\}$ is a $\mathbb{F}_{2^{-}}$-linear subspace containing exactly one half of the elements of $\mathbb{F}_{2^{r}}$. Thus $\left\{u^{2}+a_{5} u: u \in \mathbb{F}_{2^{r}}\right\}$ contains either $a_{4}$ or $a_{4}+\omega\left(a_{5}\right)$ (but not both). Take $u \in \mathbb{F}_{2^{r}}$ such that $u^{2}+a_{5} u \in\left\{a_{4}, a_{4}+\omega\left(a_{5}\right)\right\}$, then $f(x+u)-f(u)$ satisfies (R2).

(R3): Suppose $a_{7}=a_{6}=0 \neq a_{5}$. If $a_{4} \neq 0$, we can replace $f(x)=x^{8}+\sum_{i=1}^{5} a_{i} x^{i}$ by

$$
f(x+u)-f(u)=x^{8}+a_{5} x^{5}+\left(a_{5} u+a_{4}\right) x^{4}+\text { lower terms, }
$$


with $u=a_{5}^{-1} a_{4}$ annihilating the coefficient of $x^{4}$. Hereafter assume $a_{4}=0$, and replace $f$ by

$$
t^{-8} f(t x)=x^{8}+t^{-3} a_{5} x^{5}+\sum_{i=1}^{3} t^{i-8} a_{i} x^{i}
$$

with an arbitrary $t \in \mathbb{F}_{2^{r}}^{*}$. Let

$$
\Lambda=\left\{e^{i}: 0 \leqslant i<\operatorname{gcd}\left(3,2^{r}-1\right)\right\}= \begin{cases}\{1\} & \text { if } r \text { is odd } \\ \left\{1, e, e^{2}\right\} & \text { if } r \text { is even }\end{cases}
$$

which is a complete set of coset representatives of $\mathbb{F}_{2^{r}}^{*} /\left\{t^{3}: t \in \mathbb{F}_{2^{r}}^{*}\right\}$. Certain $t \in \mathbb{F}_{2^{r}}^{*}$ ensures that $t^{-3} a_{5} \in \Lambda$, and thus $t^{-8} f(t x)$ satisfies (R3).

So far we have showed that each polynomial of degree 8 over $\mathbb{F}_{2^{r}}$ is linearly ralated to some $f(x)=x^{8}+\sum_{i=1}^{7} a_{i} x^{i} \in \mathbb{F}_{2^{r}}[x]$ with all $a_{i} \in \mathbb{F}_{2^{r}}$ satisfying $(\mathbf{R 1}) \sim(\mathbf{R 3})$. In the following, suppose $\left(a_{7}, a_{6}, a_{5}\right) \neq(0,0,0)$ and let $f(x)$ be linearly related to $g(x)=x^{8}+\sum_{i=1}^{7} a_{i}^{\prime} x^{i} \in \mathbb{F}_{2^{r}}[x]$ with all $a_{i}^{\prime} \in \mathbb{F}_{2^{r}}$ satisfying $(\mathbf{R 1}) \sim(\mathbf{R 3})$. By definition, there exist $s, t \in \mathbb{F}_{2^{r}}^{*}$ and $u, v \in \mathbb{F}_{2^{r}}$ such that

$$
\begin{aligned}
x^{8}+\sum_{i=1}^{7} a_{i}^{\prime} x^{i} & =g(x)=s f(t x+u)+v \\
& =s t^{8} x^{8}+s t^{7} a_{7} x^{7}+s\left(a_{7} u+a_{6}\right) t^{6} x^{6}+\text { lower terms. }
\end{aligned}
$$

Comparing the coefficients of $x^{8}$, we see that $s=t^{-8}$.

(1) Suppose $\left(a_{7}, a_{6}\right)=(1,0)$. As $a_{7}^{\prime}=s t^{7} a_{7}=t^{-1} \neq 0,\left(a_{7}^{\prime}, a_{6}^{\prime}\right)=(1,0)$ by $(\mathbf{R 1})$. So $s=t=1$. Comparing the coefficients of $x^{6}, 0=a_{6}^{\prime}=a_{7} u+a_{6}=u$. So $v=g(0)-f(u)=0$, and $f=g$.

(2) Suppose $\left(a_{7}, a_{6}\right)=(0,1)$. Note that

$$
\begin{aligned}
x^{8}+\sum_{i=1}^{7} a_{i}^{\prime} x^{i} & =g(x)=t^{-8} f(t x+u)+v \\
& =x^{8}+t^{-2} x^{6}+t^{-3} a_{5} x^{5}+t^{-4}\left(u^{2}+a_{5} u+a_{4}\right) x^{4}+\text { lower terms, }
\end{aligned}
$$

so $a_{7}^{\prime}=0 \neq a_{6}^{\prime}=t^{-2}$. By $(\mathbf{R 1}),\left(a_{7}^{\prime}, a_{6}^{\prime}\right)=(0,1)$ and $t^{2}=1$. So $t=1, a_{5}^{\prime}=a_{5}$ and $a_{4}^{\prime}-a_{4}=u^{2}+a_{5} u$. By $(\mathbf{R 2}), a_{4}^{\prime}=a_{4} \in\left\{0, \omega\left(a_{5}\right)\right\}$, and $u \in\left\{u \in \mathbb{F}_{2^{r}}: u^{2}+a_{5} u=0\right\}=\left\{0, a_{5}\right\}$. If $u=0$, then $v=g(0)-f(u)=0$ and $f=g$. Hereafter assume $u=a_{5}$. Note that

$$
\begin{aligned}
& x^{8}+x^{6}+\sum_{i=1}^{5} a_{i}^{\prime} x^{i}=g(x)=f\left(x+a_{5}\right)-f\left(a_{5}\right) \\
= & x^{8}+x^{6}+a_{5} x^{5}+a_{4} x^{4}+a_{3} x^{3}+\left(a_{2}+a_{3} a_{5}+a_{5}^{4}\right) x^{2}+\left(a_{1}+a_{3} a_{5}^{2}+a_{5}^{5}\right) x .
\end{aligned}
$$

So $\left(a_{7}^{\prime}, a_{6}^{\prime}, a_{5}^{\prime}, a_{4}^{\prime}, a_{3}^{\prime}, a_{2}^{\prime}, a_{1}^{\prime}\right)=\left(a_{7}, a_{6}, a_{5}, a_{4}, a_{3}, a_{2}+a_{3} a_{5}+a_{5}^{4}, a_{1}+a_{3} a_{5}^{2}+a_{5}^{5}\right)$. In this case, $f \neq g$ if and only if $a_{5}\left(a_{3}+a_{5}^{3}\right) \neq 0$.

(3) Suppose $a_{7}=a_{6}=0 \neq a_{5}$. Then $a_{4}=0$ by (R3). Note that

$$
x^{8}+\sum_{i=1}^{7} a_{i}^{\prime} x^{i}=t^{-8} f(t x+u)+v=x^{8}+t^{-3} a_{5} x^{5}+t^{-4} a_{5} u x^{4}+t^{-5} x^{3}+\text { lower terms. }
$$

So $a_{7}^{\prime}=a_{6}^{\prime}=0$, and $a_{5}^{\prime}=t^{-3} a_{5} \neq 0$. By (R3), $a_{5}=a_{5}^{\prime}=t^{-3} a_{5}$ and $0=a_{4}^{\prime}=t^{-4} a_{5} u$. Thus $t^{3}=1$ and $u=0$. Therefore, $g(x)=t^{-8} f(t x)$ with some element $t$ in the set

$$
\left\{t \in \mathbb{F}_{2^{r}}^{*}: t^{3}=1\right\}= \begin{cases}\{1\} & \text { if } r \text { is odd } \\ \left\{1, e^{\frac{2^{r}-1}{3}}, e^{\frac{2\left(2^{r}-1\right)}{3}}\right\} & \text { if } r \text { is even. }\end{cases}
$$


In this case, $f \neq g$ only when $r$ is even and $t \in\left\{e^{\frac{2^{r}-1}{3}}, e^{\frac{2\left(2^{r}-1\right)}{3}}\right\}$.

\section{HeRmite's Criterion}

Dickson $[4, \S 11]$ provided a criterion for PPs over $\mathbb{F}_{q}$ on their coefficients. It is usually called Hermite's criterion as Dickson attributed its prime field case to Hermite. Assuming some notations, we quote an explicit version of it from [11].

Let $\mathbb{N}=\{m \in \mathbb{Z}: m \geqslant 0\}$. For $m \in \mathbb{N}$ and $f \in \mathbb{F}_{q}[x]$, let $\left[x^{m}: f\right]$ denote the coefficient of $x^{m}$ in $f(x)$. In other words, for a nonzero polynomial $f \in \mathbb{F}_{q}[x]$, we have $f(x)=\sum_{m=0}^{\operatorname{deg}(f)}\left[x^{m}: f\right] \cdot x^{m}$ and $\operatorname{deg}(f)=\max \left\{m \in \mathbb{N}:\left[x^{m}: f\right] \neq 0\right\}$.

Lemma 4 (Hermite's criterion [11, Theorem 7.6]). A necessary and sufficient condition for $f \in \mathbb{F}_{q}[x]$ to be a PP over $\mathbb{F}_{q}$ is

$$
\sum_{t=1}^{\left\lfloor\frac{\operatorname{deg}\left(f^{k}\right)}{q-1}\right\rfloor}\left[x^{t(q-1)}: f^{k}\right] \begin{cases}=0 & \text { for } 1 \leqslant k \leqslant q-2(\text { and } \operatorname{gcd}(k, q)=1), \\ \neq 0 & \text { for } k=q-1 .\end{cases}
$$

Recall how to calculate $\left[x^{m}: f^{k}\right]$ with the help of multinomial coefficients. For integers $k, j_{1}$, $j_{2}, \ldots$, and $j_{t}$, define the multinomial coefficient as

$$
\left(\begin{array}{cl}
k \\
j_{1}, j_{2}, \ldots, j_{t}
\end{array}\right):= \begin{cases}\frac{k !}{j_{1} ! j_{2} ! \cdots j_{t} !} & \text { if } k=j_{1}+j_{2}+\cdots+j_{t} \text { and all } j_{1}, \ldots, j_{t} \geqslant 0 \\
0 & \text { otherwise. }\end{cases}
$$

For $f(x)=x^{8}+\sum_{i=1}^{7} a_{i} x^{i} \in \mathbb{F}_{2^{r}}[x]$ with all $a_{i} \in \mathbb{F}_{2^{r}}$, by the multinomial theorem,

$$
f(x)^{k}=\sum_{j_{1}+j_{2}+\cdots+j_{8}=k}\left(\begin{array}{c}
k \\
j_{1}, j_{2}, \ldots, j_{8}
\end{array}\right)\left(\prod_{i=1}^{7} a_{i}^{j_{i}}\right) \cdot x^{j_{1}+2 j_{2}+3 j_{3}+4 j_{4}+5 j_{5}+6 j_{6}+7 j_{7}+8 j_{8}} .
$$

Let $J(k, m)$ denote the set of all solutions $\left(j_{1}, j_{2}, \ldots, j_{8}\right) \in \mathbb{N}^{8}$ of the linear equations:

$$
\left\{\begin{aligned}
j_{1}+j_{2}+j_{3}+j_{4}+j_{5}+j_{6}+j_{7}+j_{8} & =k \\
j_{1}+2 j_{2}+3 j_{3}+4 j_{4}+5 j_{5}+6 j_{6}+7 j_{7}+8 j_{8} & =m
\end{aligned}\right.
$$

Then $\left[x^{m}: f^{k}\right]=\sum_{\left(j_{1}, \ldots, j_{8}\right) \in J(k, m)}\left(\begin{array}{c}k \\ j_{1}, j_{2}, \ldots, j_{8}\end{array}\right) a_{1}^{j_{1}} a_{2}^{j_{2}} a_{3}^{j_{3}} a_{4}^{j_{4}} a_{5}^{j_{5}} a_{6}^{j_{6}} a_{7}^{j_{7}}$.

Working over $\mathbb{F}_{2^{r}}$, the calculation of multinomial coefficients can be greatly simplified by the following multinomial analogue (and corollary) of Lucas's theorem [12, (137)].

Lemma 5. $[4, \S \S 14]$ Let $p$ be a prime, $k=\sum_{s=0}^{l} k_{s} p^{s}$, and $j_{i}=\sum_{s=0}^{l} j_{i, s} p^{s}$ for $1 \leqslant i \leqslant t$, with all $k_{s}$ and $j_{i, s} \in\{0,1,2, \ldots, p-1\}$. Then

$$
\left(\begin{array}{c}
k \\
j_{1}, j_{2}, \ldots, j_{t}
\end{array}\right) \equiv \prod_{s=0}^{l}\left(\begin{array}{c}
k_{s} \\
j_{1, s}, j_{2, s}, \ldots, j_{t, s}
\end{array}\right)(\bmod p) .
$$

In particular, $p \nmid\left(\begin{array}{c}k \\ j_{1}, j_{2}, \ldots, j_{t}\end{array}\right)$ if and only if $k_{s}=j_{1, s}+j_{2, s}+\cdots+j_{t, s}$ for any $0 \leqslant s \leqslant l$. 
For $n=\sum_{s=0}^{\left\lfloor\log _{2}(n)\right\rfloor} n_{s} 2^{s} \in \mathbb{N}$ with all $n_{s} \in\{0,1\}$, let

$$
\begin{aligned}
\beta(n) & =\left\{s \in \mathbb{N}: n_{s}=1,0 \leqslant s \leqslant \log _{2}(n)\right\} \\
& =\left\{s \in \mathbb{N}:\left\lfloor n / 2^{s}\right\rfloor \text { is odd }\right\} .
\end{aligned}
$$

When $p=2$ in Lemma 5 , note that $\left(\begin{array}{c}k \\ j_{1}, j_{2}, \ldots, j_{t}\end{array}\right) \equiv 1(\bmod 2)$ if and only if $\beta(k)$ is the disjoint union $\bigsqcup_{i=1}^{t} \beta\left(j_{i}\right)$ of its subsets $\beta\left(j_{1}\right), \beta\left(j_{2}\right) \ldots, \beta\left(j_{t}\right)$ (i.e. $\beta(k)=\bigcup_{i=1}^{t} \beta\left(j_{i}\right)$ and $\beta\left(j_{i}\right) \cap \beta\left(j_{i^{\prime}}\right)=\varnothing$ for any $\left.i \neq i^{\prime}\right)$. Therefore,

$$
\left[x^{m}: f^{k}\right]=\sum_{\substack{\beta(k)=\bigsqcup_{i=1}^{8} \beta\left(j_{i}\right) \\ j_{1}+2 j_{2}+3 j_{3}+\cdots+8 j_{8}=m}} a_{1}^{j_{1}} a_{2}^{j_{2}} a_{3}^{j_{3}} a_{4}^{j_{4}} a_{5}^{j_{5}} a_{6}^{j_{6}} a_{7}^{j_{7}} \in \mathbb{F}_{2^{r}} .
$$

Suppose $\beta(k)=\left\{s_{0}, s_{1}, \ldots, s_{n-1}\right\}$, then the set $\left\{\left(j_{1}, j_{2}, \ldots, j_{8}\right) \in \mathbb{N}^{8}: \beta(k)=\bigsqcup_{i=1}^{8} \beta\left(j_{i}\right)\right\}$ contains exactly $8^{n}$ elements, which can be listed explicitly by the one-to-one correspondence:

$$
\begin{aligned}
& \left\{\left(j_{1}, j_{2}, \ldots, j_{8}\right) \in \mathbb{N}^{8}: \beta(k)=\bigsqcup_{i=1}^{8} \beta\left(j_{i}\right)\right\} \stackrel{\simeq}{\longleftrightarrow}\left\{0,1,2,3, \ldots, 8^{n}-1\right\} . \\
& \left(j_{1}, j_{2}, \ldots, j_{8}\right) \text { with } \leftrightarrow \quad u=\sum_{v=0}^{n-1} u_{v} 8^{v} \text { with } \\
& j_{i}=\left\{\begin{array}{ll}
\sum_{0 \leqslant v<n} 2^{s_{v}} & \text { if } 1 \leqslant i \leqslant 7 \\
\sum_{\substack{0 \leqslant v<n \\
u_{v}=0}} 2^{s_{v}} & \text { if } i=8
\end{array} \quad u_{v}= \begin{cases}i & \text { if } s_{v} \in \beta\left(j_{i}\right) \text { and } 1 \leqslant i \leqslant 7 \\
0 & \text { if } s_{v} \in \beta\left(j_{8}\right)\end{cases} \right.
\end{aligned}
$$

By the above arguments, we can calculate the left hand side in Lemma 4 (Hermite's criterion) for the $k$-th power of a $\operatorname{PP} f(x)=x^{8}+\sum_{i=1}^{7} a_{i} x^{i}$ over $\mathbb{F}_{q}\left(\right.$ with $\left.q=2^{r}\right)$ as

$$
\begin{aligned}
& \mathbf{H C}\left(r, k, a_{7}, a_{6}, a_{5}, a_{4}, a_{3}, a_{2}, a_{1}\right)=\sum_{\substack{t=1 \\
0 \leqslant u<8^{n}}}^{\left\lfloor\frac{8 k}{q-1}\right\rfloor}\left[x^{t(q-1)}: f^{k}\right] \\
= & a_{1}^{j_{1}(u)} a_{2}^{j_{2}(u)} a_{3}^{j_{3}(u)} a_{4}^{j_{4}(u)} a_{5}^{j_{5}(u)} a_{6}^{j_{6}(u)} a_{7}^{j_{7}(u)}, \\
& \sum_{i=1}^{7} i j_{i}(u)+8 j_{0}(u) \in\left\{t(q-1): 1 \leqslant t \leqslant\left\lfloor\frac{8 k}{q-1}\right\rfloor\right\}
\end{aligned}
$$

with $j_{i}(u)=\sum_{\substack{0 \leqslant v<n \\ u_{v}=i}} 2^{s_{v}}$ for $0 \leqslant i \leqslant 7$, where $\left\{s_{0}, s_{1}, \ldots, s_{n-1}\right\}=\beta(k)$, and $u=\sum_{v=0}^{n-1} u_{v} 8^{v}$ with all $u_{v} \in\{0,1,2, \ldots, 7\}$.

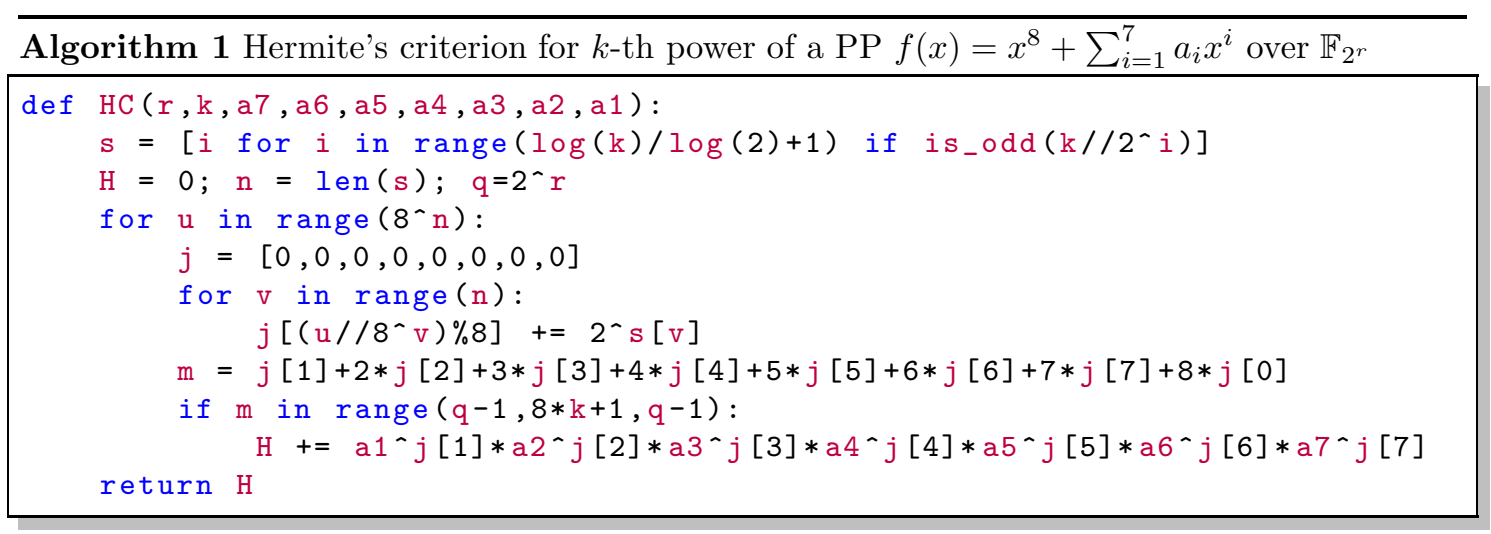


Algorithm 1 realizes $\mathbf{H C}\left(r, k, a_{7}, a_{6}, a_{5}, a_{4}, a_{3}, a_{2}, a_{1}\right)$ as a SageMath function. When running it, we can either input some explicit values of $a_{i}$, or input the indeterminate $a_{i}$ itself as working over the polynomial ring $\mathbb{F}_{2}\left[a_{1}, a_{2}, a_{3}, a_{4}, a_{5}, a_{6}, a_{7}\right]$. For example, we can calculate $\mathbf{H C}\left(5,27, a_{7}, 0, a_{5}, 1, a_{3}, a_{2}, a_{1}\right)$ by the following SageMath codes:

K.<a1, a2, a3, a4, a5, a6, a $>$ = PolynomialRing (GF(2))

HC $(5,27, a 7,0, a 5,1, a 3, a 2$, a 1$)$

By Lemma 4 (Hermite's criterion), an output of $\mathbf{H C}$ for specifice $r$ and $k$ (such that $1 \leqslant k \leqslant$ $2^{r}-2$ ) provides an explicit equation satisfied by the polynomial coefficients of a PP over $\mathbb{F}_{2^{r}}$.

\section{Case by Case}

In this section, we analysis non-exceptional PPs over $\mathbb{F}_{2^{r}}$ of the form $f(x)=x^{8}+\sum_{i=1}^{7} a_{i} x^{i}$ with all $a_{i} \in \mathbb{F}_{2^{r}}$ subject to requirements $(\mathbf{R} 1) \sim(\mathbf{R 3})$ of Proposition 3, on a case-by-case basis for $r \in\{4,5,6,7,8,9\}$, in light of the aforementioned equations

$$
\mathbf{H C}\left(r, k, a_{7}, a_{6}, a_{5}, a_{4}, a_{3}, a_{2}, a_{1}\right)=0
$$

with $1 \leqslant k \leqslant 2^{r}-2$, from outputs of Algorithm 1 running on SageMath 8.6.

For later use, we define a SageMath function $\mathbf{P P}\left(q, e, a_{7}, a_{6}, a_{5}, a_{4}, a_{3}, a_{2}, a_{1}\right)$ in Algorithm 2 to examine whether $f(x)=x^{8}+\sum_{i=1}^{7} a_{i} x^{i}$ is a PP over $\mathbb{F}_{q}$. The following Lemma 6 ensures that it suffices to check whether the values $f\left(e^{j}\right)$ for $0 \leqslant j \leqslant\left\lfloor q-\frac{q-1}{8}\right\rfloor$ are distinct, provided a generator $e$ of the multiplicative group $\mathbb{F}_{q}^{*}$.

Lemma 6 (Wan [15]). A polynomial $f \in \mathbb{F}_{q}[x]$ of degree $n \geqslant 1$ is a PP over $\mathbb{F}_{q}$ if its value set $\left\{f(c): c \in \mathbb{F}_{q}\right\}$ contains at least $\left\lfloor q-\frac{q-1}{n}\right\rfloor+1$ distinct values.

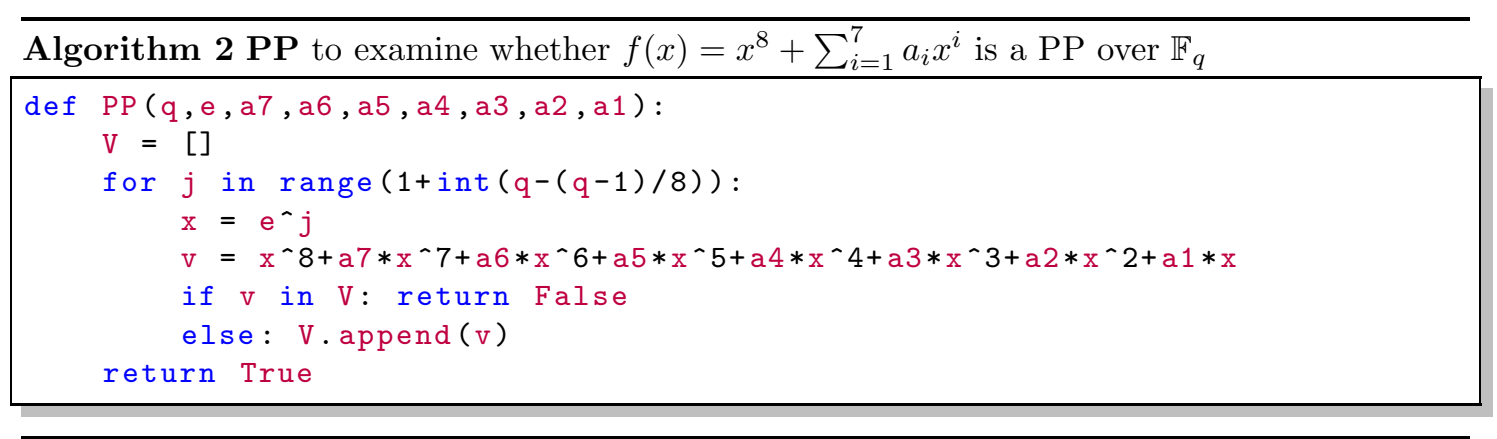

- Note that $\left(a_{7}, a_{6}, a_{5}, a_{3}\right) \neq(0,0,0,0)$ for $f$ to be a non-exceptional PP over $\mathbb{F}_{2^{r}}$.

- Let $e$ be a generator of the multiplicative group $\mathbb{F}_{2^{r}}^{*}$.

- For each $a \in \mathbb{F}_{2^{r}}^{*}$, fix an element $\omega(a)$ in the set $\mathbb{F}_{2^{r}} \backslash\left\{u^{2}+a u: u \in \mathbb{F}_{2^{r}}\right\}$.

4.1. Case $r=4, q=16$. Note that

$$
\begin{aligned}
& 0=\mathbf{H C}\left(4,3, a_{7}, a_{6}, a_{5}, a_{4}, a_{3}, a_{2}, a_{1}\right)=a_{5}^{3}+a_{3} a_{6}^{2}+a_{4}^{2} a_{7}+a_{1} a_{7}^{2}, \\
& 0=\mathbf{H C}\left(4,5, a_{7}, a_{6}, a_{5}, a_{4}, a_{3}, a_{2}, a_{1}\right)=a_{3}^{5}+a_{6}^{5}+a_{2}^{4} a_{7}+a_{2} a_{7}^{4} .
\end{aligned}
$$

If $a_{7}=a_{6}=0$, then $a_{5}=a_{3}=0$. As $\left(a_{7}, a_{6}, a_{5}, a_{3}\right) \neq(0,0,0,0)$, we have $\left(a_{7}, a_{6}\right) \neq(0,0)$. By $(\mathbf{R} 1),\left(a_{7}, a_{6}\right)=(1,0)$ or $(0,1)$. 
(1) Suppose $\left(a_{7}, a_{6}\right)=(1,0)$. Then $a_{1}=a_{5}^{3}+a_{4}^{2}$ and $a_{3}^{5}=a_{2}^{4}+a_{2}$.

(2) Suppose $\left(a_{7}, a_{6}\right)=(0,1)$. Then $a_{3}^{5}=1$ and $a_{3}=a_{5}^{3} \neq 0$. By $(\mathbf{R 2}), a_{4} \in\left\{0, \omega\left(a_{5}\right)\right\}$.

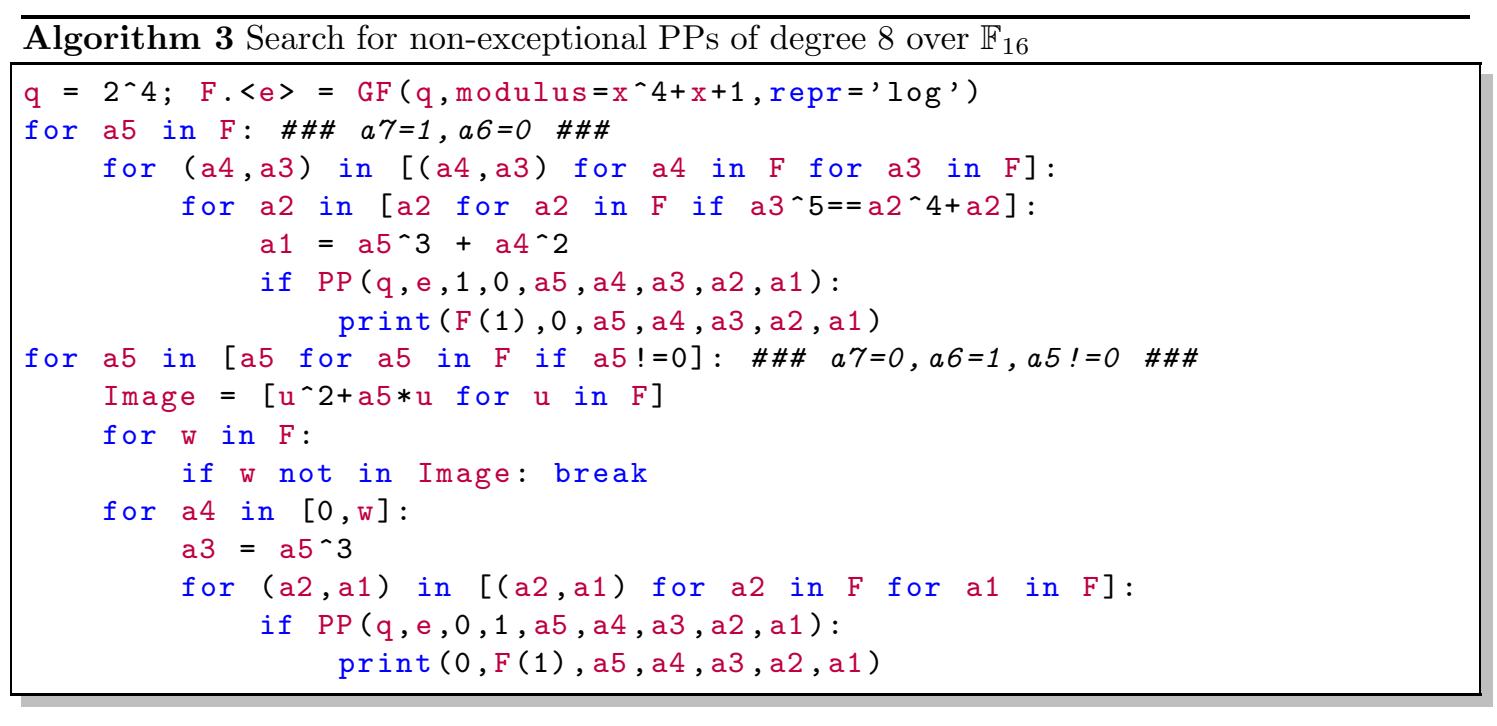

Therefore, we write Algorithm 3 to search for all non-exceptional PPs of degree 8 over $\mathbb{F}_{16}$ up to linear transformations, and run it in SageMath 8.6.

- In Algorithm 3, we take $e$ as a root of the Conway polynomial ${ }^{1} x^{4}+x+1$ in $\mathbb{F}_{16}$, which is a generator of the multiplicative group $\mathbb{F}_{16}^{*}$. In the outputs, an element $e^{i}$ of $\mathbb{F}_{16}$ is represented by the integer $i$ such that $1 \leqslant i \leqslant q-1$, while the zero element of $\mathbb{F}_{16}$ is represented by 0 .

- The outputs of Algorithm 3 are 113 tuples of the form $\left(0,1, a_{5}, a_{4}, a_{3}, a_{2}, a_{1}\right)$ such that $a_{3}=a_{5}^{3} \neq 0$. By Proposition 3 , they are not linearly related to each other, and correspond to all 113 linearly related classes of non-exceptional PPs of degree 8 over $\mathbb{F}_{16}$.

To save space, we will write down the result up to a composition of the Frobenius automorphism $\left(\mathbb{F}_{2^{r}} \rightarrow \mathbb{F}_{2^{r}}\right.$ with $\left.a \mapsto a^{2}\right)$ with itself, as the following Proposition 7 indicates.

Proposition 7. For $1 \leqslant r \in \mathbb{Z}$, let $\Gamma(r)$ be a subset of $\mathbb{F}_{2^{r}}$ such that

$$
\mathbb{F}_{2^{r}}^{*}=\left\{a^{2^{j}}: a \in \Gamma(r), 0 \leqslant j \leqslant r-1\right\} .
$$

For each $a \in \Gamma(r)$, fix an element $\omega(a)$ in the set $\mathbb{F}_{2^{r}} \backslash\left\{u^{2}+a u: u \in \mathbb{F}_{2^{r}}\right\}$. Let $h(x)=$ $x^{8}+x^{6}+\sum_{i=1}^{5} c_{i} x^{i} \in \mathbb{F}_{2^{r}}[x]$ with all $c_{i} \in \mathbb{F}_{2^{r}}$ and $c_{5} \neq 0$. Then $h(x)$ is linearly related to some $x^{8}+x^{6}+\sum_{i=1}^{5} a_{i}^{2^{j}} x^{i} \in \mathbb{F}_{2^{r}}[x]$ with $0 \leqslant j \leqslant r-1, a_{5} \in \Gamma(r), a_{4} \in\{0, \omega(a)\}$ and all $a_{i} \in \mathbb{F}_{2^{r}}$. Moreover, $h$ is a (non-exceptional) PP over $\mathbb{F}_{2^{r}}$ if and only if so is $x^{8}+x^{6}+\sum_{i=1}^{5} a_{i} x^{i}$.

\footnotetext{
${ }^{1}$ The Conway polynomial $C_{p, n}$ is a particular irreducible polynomial of degree $n$ over $\mathbb{F}_{p}$ named after John H. Conway by Richard A. Parker, satisfying a certain compatibility condition proposed by Conway. The Conway polynomial is chosen to be primitive, so that each of its roots generates the multiplicative group $\mathbb{F}_{p^{n}}$. See its wikipedia page and Frank Lübeck's webpage "Conway polynomials for finite fields for more information.
} 
Proof. By definition, there exist $a_{5} \in \Gamma(r)$ and $0 \leqslant j \leqslant r-1$ such that $c_{5}=a_{5}^{2^{j}}$. Let

$$
\varphi(x)=x^{8}+x^{6}+a_{5} x^{5}+\sum_{i=1}^{4} c_{i}^{2^{r-j}} x^{i} .
$$

By the same arguments for $(\mathbf{R 2})$ of Proposition $3, \varphi(x)$ is linearly related to some $f(x)=$ $x^{8}+x^{6}+a_{5} x^{5}+\sum_{i=1}^{4} a_{i} x^{i}$ with all $a_{i} \in \mathbb{F}_{2^{r}}$ and $a_{4} \in\{0, \omega(a)\}$. Then $h(x)$ is linearly related to $g(x)=x^{8}+x^{6}+a_{5}^{2^{j}} x^{5}+\sum_{i=1}^{4} a_{i}^{2^{j}} x^{i}$. For $1 \leqslant m \in \mathbb{Z}$, as $\mathbb{F}_{2^{r m}} \rightarrow \mathbb{F}_{2^{r m}}\left(a \mapsto a^{2^{j}}\right)$ is a field automorphism, $h$ permutes $\mathbb{F}_{2^{r m}} \Leftrightarrow \varphi$ permutes $\mathbb{F}_{2^{r m}} \Leftrightarrow f$ permutes $\mathbb{F}_{2^{r m}} \Leftrightarrow g$ permutes $\mathbb{F}_{2^{r m}}$.

Let us take $\Gamma(4)=\left\{1, e, e^{3}, e^{5}, e^{7}\right\}$. By Proposition 7, up to a composition of the Frobenius automorphism with itself, we only need to pick up outputting tuples $\left(0,1, a_{5}, a_{4}, a_{3}, a_{2}, a_{1}\right)$ such that $a_{5} \in\left\{1, e, e^{3}, e^{5}, e^{7}\right\}$, which gives the following theorem.

Theorem 8. Let e be a root of the Conway polynomial $x^{4}+x+1$ in $\mathbb{F}_{16}$. Each non-exceptional $P P$ of degree 8 over $\mathbb{F}_{16}$ is linearly related to a polynomial of the form $x^{8}+x^{6}+\sum_{i=1}^{5} a_{i}^{2^{j}} x^{i}$, with $j \in\{0,1,2,3\}$ and $\left(a_{5}, a_{4}, a_{3}, a_{2}, a_{1}\right) \in \mathbb{F}_{16}^{5}$ in the following list:

$$
\begin{array}{llll}
\left(e, 0, e^{3}, e^{5}, e\right), & \left(e, 0, e^{3}, e^{9}, e^{10}\right), & \left(e, e, e^{3}, e^{8}, e^{11}\right), & \left(e, e, e^{3}, e^{9}, e^{12}\right), \\
\left(e, e, e^{3}, e^{11}, e^{4}\right), & \left(e, e, e^{3}, e^{12}, e^{9}\right), & \left(e, e, e^{3}, e^{13}, e^{4}\right), & \left(e^{3}, 0, e^{9}, e^{5}, 1\right), \\
\left(e^{3}, 0, e^{9}, e^{8}, e^{8}\right), & \left(e^{3}, e^{2}, e^{9}, 0, e^{12}\right), & \left(e^{3}, e^{2}, e^{9}, e^{7}, e^{12}\right), & \left(e^{3}, e^{2}, e^{9}, 1, e^{11}\right), \\
\left(e^{5}, 0,1, e^{3}, 1\right), & \left(e^{5}, 0,1, e^{10}, e\right), & \left(e^{5}, 0,1, e^{10}, e^{4}\right), & \left(e^{5}, 0,1, e^{12}, 1\right), \\
\left(e^{5}, e, 1,0, e^{13}\right), & \left(e^{5}, e, 1,0, e^{14}\right), & \left(e^{5}, e, 1, e^{5}, e^{3}\right), & \left(e^{5}, e, 1, e^{5}, e^{13}\right), \\
\left(e^{5}, e, 1, e^{7}, e^{7}\right), & \left(e^{5}, e, 1, e^{9}, e^{6}\right), & \left(e^{5}, e, 1, e^{9}, e^{7}\right), & \left(e^{7}, 0, e^{6}, e, e^{7}\right), \\
\left(e^{7}, 0, e^{6}, e^{2}, e^{5}\right), & \left(e^{7}, 0, e^{6}, e^{9}, e^{7}\right), & \left(e^{7}, 0, e^{6}, e^{9}, e^{14}\right), & \left(e^{7}, 0, e^{6}, e^{11}, e^{6}\right), \\
\left(e^{7}, 0, e^{6}, e^{12}, e^{2}\right), & \left(e^{7}, e^{2}, e^{6}, e, e^{9}\right), & \left(e^{7}, e^{2}, e^{6}, e^{5}, e\right), & \left(e^{7}, e^{2}, e^{6}, e^{12}, e^{10}\right), \\
(1,0,1, e, 1), & \left(1, e^{3}, 1, e, e^{13}\right), & \left(1, e^{3}, 1, e^{2}, e^{13}\right) . &
\end{array}
$$

Remark. Outside the list of Theorem 8 are there four other outputting tuples of Algorithm 3 with $a_{5} \in \Gamma(4)=\left\{1, e, e^{3}, e^{5}, e^{7}\right\}$ :

$$
\left(1,0,1, e^{2}, 1\right), \quad\left(1,0,1, e^{4}, 1\right), \quad\left(1,0,1, e^{8}, 1\right), \quad\left(1, e^{3}, 1, e^{4}, e^{13}\right) .
$$

The first three tuples are clearly equivalent to $(1,0,1, e, 1)$ up to a composition of the Frobenius automorphism with itself. The last tuple gives $g(x)=x^{8}+x^{6}+x^{5}+e^{3} x^{4}+x^{3}+e^{4} x^{2}+e^{13} x$, linearly related to $g\left(x+e_{3}\right)+e^{13}=x^{8}+x^{6}+x^{5}+e^{6} x^{4}+x^{3}+e^{2} x^{2}+e^{26} x$, corresponding to the tuple $\left(1, e^{6}, 1, e^{2}, e^{26}\right)$ equivalent to $\left(1, e^{3}, 1, e, e^{13}\right)$ up to the Frobenius automorphism. The list of Theorem 8 for $r=4$ is actually complete and non-repetitive up to compositions of linear transformations and Frobenius automorphisms.

4.2. Case $r=5, q=32$. As $\mathbf{H C}\left(5,7,0,0,0, a_{4}, a_{3}, a_{2}, a_{1}\right)=a_{3}^{5}$, so $a_{3}=0$ if $a_{7}=a_{6}=a_{5}=0$. As $\left(a_{7}, a_{6}, a_{5}, a_{3}\right) \neq(0,0,0,0),\left(a_{7}, a_{6}, a_{5}\right) \neq(0,0,0)$. By $(\mathbf{R} 1),\left(a_{7}, a_{6}\right)=(0,0),(1,0)$ or $(0,1)$.

(1) Suppose $a_{7}=a_{6}=0 \neq a_{5}$. By (R3), $a_{5}=1$ and $a_{4}=0$. We have $a_{1}=a_{3}^{5}+a_{3}^{2}$ as

$$
0=\mathbf{H C}\left(5,7,0,0,1,0, a_{3}, a_{2}, a_{1}\right)=a_{3}^{5}+a_{3}^{2}+a_{1} .
$$

(2) Suppose $\left(a_{7}, a_{6}\right)=(1,0)$. Then $\mathbf{H C}\left(5,5,1,0, a_{5}, a_{4}, a_{3}, a_{2}, a_{1}\right)=a_{3}=0$.

(3) Suppose $\left(a_{7}, a_{6}\right)=(0,1)$. By (R2), $a_{4} \in\left\{0, \omega\left(a_{5}\right)\right\}$ if $a_{5} \neq 0 ; a_{4}=0$ if $a_{5}=0$. 


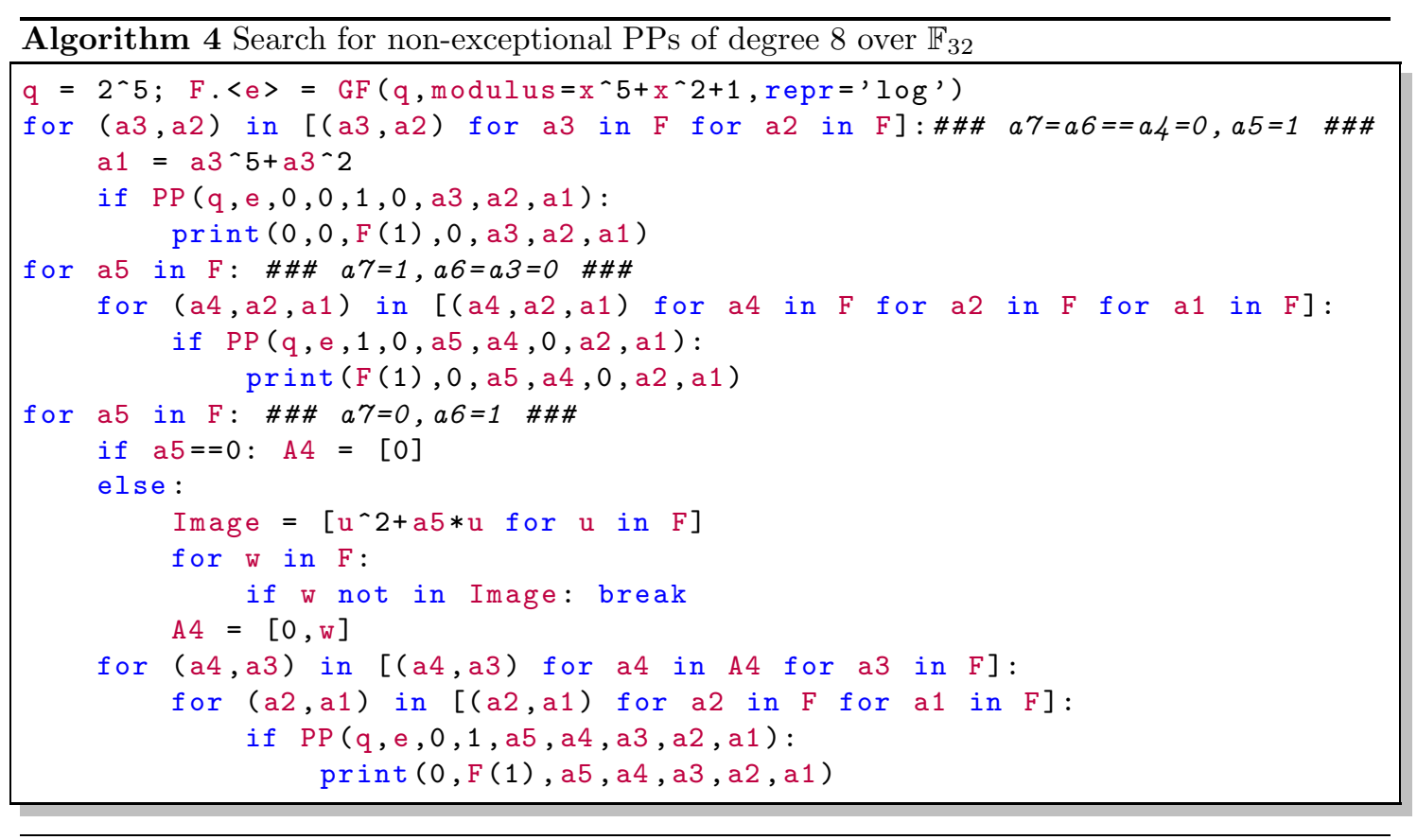

Therefore, we write Algorithm 4 to search for all non-exceptional PPs of degree 8 over $\mathbb{F}_{32}$ up to linear transformations, and run it in SageMath 8.6.

- The outputs of Algorithm 4 are 20 tuples of the form $\left(0,1, a_{5}, a_{4}, a_{3}, a_{2}, a_{1}\right)$ such that $a_{5}\left(a_{3}+a_{5}^{3}\right) \neq 0$. By Proposition 3 , they are linearly related into exactly 10 pairs, which correspond to all 10 linearly related classes of non-exceptional PPs of degree 8 over $\mathbb{F}_{32}$.

- In the outputs of Algorithm $4, a_{5} \in\left\{e, e^{2}, e^{4}, e^{8}, e^{11}, e^{13}, e^{16}, e^{21}, e^{22}, e^{26}\right\}$. By Proposition 7, up to a composition of the Frobenius automorphism with itself, we only need to pick up outputting tuples $\left(0,1, a_{5}, a_{4}, a_{3}, a_{2}, a_{1}\right)$ such that $a_{5} \in\left\{e, e^{11}\right\}$.

Theorem 9. Let e be a root of the Conway polynomial $x^{5}+x^{2}+1$ in $\mathbb{F}_{32}$. Each non-exceptional PP of degree 8 over $\mathbb{F}_{32}$ is linearly related to one of the following:

$$
\begin{gathered}
x^{8}+x^{6}+e^{2^{j}} x^{5}+e^{26 \cdot 2^{j}} x^{3}+e^{25 \cdot 2^{j}} x^{2}, \\
x^{8}+x^{6}+e^{11 \cdot 2^{j}} x^{5}+e^{2^{j}} x^{4}+e^{29 \cdot 2^{j}} x^{3}+e^{27 \cdot 2^{j}} x,
\end{gathered}
$$

with $j$ running through the set $\{0,1,2,3,4\}$.

4.3. Case $r=6, q=64$. First, $a_{7}=0$ as $\mathbf{H C}\left(6,9, a_{7}, a_{6}, a_{5}, a_{4}, a_{3}, a_{2}, a_{1}\right)=a_{7}^{9}$. Note that

$$
\begin{aligned}
& 0=\mathbf{H C}\left(6,11,0, a_{6}, a_{5}, a_{4}, a_{3}, a_{2}, a_{1}\right)=a_{5}^{3} a_{6}^{8}+a_{3} a_{6}^{10}=a_{6}^{8}\left(a_{5}^{3}+a_{3} a_{6}^{2}\right), \\
& 0=\mathbf{H C}\left(6,21,0, a_{6}, a_{5}, a_{4}, a_{3}, a_{2}, a_{1}\right)=a_{3}^{21}+a_{6}^{21} .
\end{aligned}
$$

Note the relations: $a_{3} \neq 0 \Leftrightarrow a_{6} \neq 0 \Rightarrow a_{5}^{3}=a_{3} a_{6}^{2} \neq 0$. Recall that $\left(a_{6}, a_{5}, a_{3}\right) \neq(0,0,0)$. If $a_{6}=0$, then $a_{3}=0 \neq a_{5}$. In any case, we have $a_{5} \neq 0$. By $(\mathbf{R} 1),\left(a_{7}, a_{6}\right)=(0,0)$, or $(0,1)$.

(1) Suppose $\left(a_{7}, a_{6}\right)=(0,0)$. Already $a_{3}=0 \neq a_{5}$. By (R3), $a_{4}=0$ and $a_{5} \in\left\{1, e, e^{2}\right\}$.

(2) Suppose $\left(a_{7}, a_{6}\right)=(0,1)$. As mentioned above, $a_{3}=a_{5}^{3} \neq 0$. By $(\mathbf{R 2}), a_{4} \in\left\{0, \omega\left(a_{5}\right)\right\}$. 


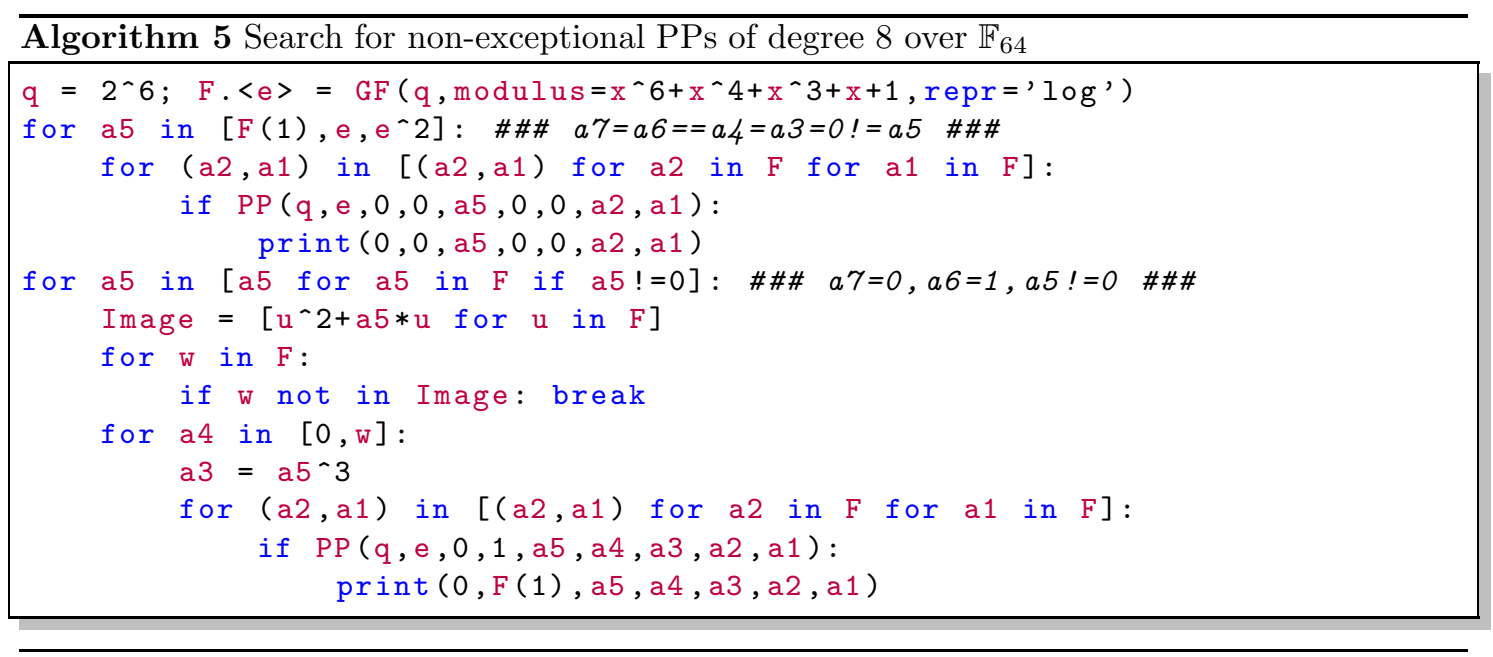

Therefore, we write Algorithm 5 to search for all non-exceptional PPs of degree 8 over $\mathbb{F}_{64}$ up to linear transformations, and run it in SageMath 8.6. The outputs can be reworded as the following theorem.

Theorem 10. Let $e$ be a root of the Conway polynomial $x^{6}+x^{4}+x^{3}+x+1$ in $\mathbb{F}_{64}$. Each non-exceptional PP of degree 8 over $\mathbb{F}_{64}$ is linearly related to one of the following:

$$
x^{8}+e x^{5}+e^{2} x^{2}, \quad x^{8}+e^{2} x^{5}+e^{4} x^{2}, \quad x^{8}+x^{6}+x^{5}+e^{3} x^{4}+x^{3}+e^{14} x^{2}+e^{6} x .
$$

4.4. Case $r=7, q=128$.

Theorem 11. All PPs of degree 8 over $\mathbb{F}_{128}$ are exceptional.

Proof. Let us prove it by reduction to absurdity. Suppose that $f$ is a non-exceptional PP of degree 8 over $\mathbb{F}_{128}$. Without loss of generality, we can assume that $f(x)=x^{8}+\sum_{i=1}^{7} a_{i} x^{i}$ with all $a_{i} \in \mathbb{F}_{128}$ satisfying requirements $(\mathbf{R 1})$ and (R2) of Proposition 3. As we mentioned in the introduction section, a linearized PP must be exceptional. In particular, we note that:

- $\left(a_{7}, a_{6}, a_{5}, a_{3}\right) \neq(0,0,0,0)$;

- $\left(a_{7}, a_{6}\right) \in\{(1,0),(0,1),(0,0)\}$;

- if $\left(a_{7}, a_{6}, a_{5}\right)=(0,1,0)$ then $a_{4}=0$.

Note that

$$
\begin{aligned}
& \mathbf{H C}\left(7,23,0,0, a_{5}, a_{4}, a_{3}, a_{2}, a_{1}\right)=a_{5}^{19}, \\
& \mathbf{H C}\left(7,29,0,0,0, a_{4}, a_{3}, a_{2}, a_{1}\right)=a_{3}^{21} .
\end{aligned}
$$

If $a_{7}=a_{6}=0$, then $a_{5}=a_{3}=0$. So $\left(a_{7}, a_{6}\right) \neq(0,0)$. Thus $\left(a_{7}, a_{6}\right)=(0,1)$ or $(1,0)$.

(1) Suppose $\left(a_{7}, a_{6}, a_{5}\right)=(0,1,0)$. Then $a_{4}=0$. Note that

$$
\begin{aligned}
& \mathbf{H C}\left(7,43,0,1,0,0,0, a_{2}, a_{1}\right)=a_{2}, \\
& \mathbf{H C}\left(7,55,0,1,0,0,0,0, a_{1}\right)=a_{1}^{16} .
\end{aligned}
$$


If $a_{3}=0$, then $a_{2}=a_{1}=0$, and $f(x)=x^{8}+x^{6}$ is not a PP. So $a_{3} \neq 0$. Also note that

$$
\begin{aligned}
0 & =\mathbf{H C}\left(7,23,0,1,0,0, a_{3}, a_{2}, a_{1}\right)=a_{3}^{5}+a_{2}^{2} a_{3}+a_{1} a_{3}^{2}, \\
0 & =\mathbf{H C}\left(7,27,0,1,0,0, a_{3}, a_{2}, a_{1}\right)=a_{3}^{17}+a_{2}^{2} a_{3}^{9}+a_{1} a_{3}^{10}+a_{2}^{8} a_{3}, \\
0 & =\mathbf{H C}\left(7,23,0,1,0,0, a_{3}, a_{2}, a_{1}\right) \cdot a_{3}^{8}+\mathbf{H C}\left(7,27,0,1,0,0, a_{3}, a_{2}, a_{1}\right) \\
& =a_{3}^{17}+a_{3}^{13}+a_{2}^{8} a_{3}=a_{3}\left(a_{3}^{4}+a_{3}^{3}+a_{2}^{2}\right)^{4} .
\end{aligned}
$$

Let $a_{3}=t^{2}$ with $t \in \mathbb{F}_{128}^{*}$. Then $a_{2}=t^{4}+t^{3}, a_{1}=a_{3}^{3}+a_{2}^{2} a_{3}^{-1}=t^{4}$, and

$$
f(x)=x^{8}+x^{6}+t^{2} x^{3}+\left(t^{4}+t^{3}\right) x^{2}+t^{4} x .
$$

Note that $f(t+1)=t^{8}+t^{5}=f(t)$. So $f$ is not a PP over $\mathbb{F}_{128}$.

(2) Suppose $\left(a_{7}, a_{6}\right)=(0,1)$ and $a_{5} \neq 0$. Note that

$$
\begin{aligned}
0 & =\mathbf{H C}\left(7,23,0,1, a_{5}, a_{4}, a_{3}, a_{2}, a_{1}\right)^{4} \cdot a_{3}+\mathbf{H C}\left(7,29,0,1, a_{5}, a_{4}, a_{3}, a_{2}, a_{1}\right) \\
& =a_{3} a_{5}^{76}+a_{3}^{5} a_{5}^{64}=a_{3} a_{5}^{64}\left(a_{5}^{3}+a_{3}\right)^{4} .
\end{aligned}
$$

As $a_{5} \neq 0$, we have $a_{3} \in\left\{0, a_{5}^{3}\right\}$.

- Suppose $a_{3}=0$. Note that

$$
\begin{aligned}
0 & =\mathbf{H C}\left(7,27,0,1, a_{5}, a_{4}, 0, a_{2}, a_{1}\right)+\mathbf{H C}\left(7,43,0,1, a_{5}, a_{4}, 0, a_{2}, a_{1}\right)^{8} \cdot a_{5}^{3} \\
& =a_{2}^{256} a_{5}^{27}+a_{5}^{67}+a_{1}^{2} a_{5}^{25}=a_{2}^{2} a_{5}^{27}+a_{5}^{67}+a_{1}^{2} a_{5}^{25} .
\end{aligned}
$$

So $a_{1}=a_{5}^{21}+a_{2} a_{5}$. Also note that

$$
\begin{aligned}
0 & =\mathbf{H C}\left(7,23,0,1, a_{5}, a_{4}, 0, a_{2}, a_{5}^{21}+a_{2} a_{5}\right) \\
& =a_{5}^{43}+a_{5}^{27}+a_{5}^{19}+a_{2} a_{5}^{7}+a_{4}^{4} a_{5}^{3}+a_{2}^{2} a_{5}^{3} .
\end{aligned}
$$

So $a_{4}=a_{2}^{64}+a_{2}^{32} a_{5}+a_{5}^{10}+a_{5}^{6}+a_{5}^{4}$. Then

$$
\begin{aligned}
0 & =\mathbf{H C}\left(7,27,0,1, a_{5}, a_{2}^{64}+a_{2}^{32} a_{5}+a_{5}^{10}+a_{5}^{6}+a_{5}^{4}, 0, a_{2}, a_{5}^{21}+a_{2} a_{5}\right) \\
& =a_{2}^{1024} a_{5}^{3}+a_{2}^{256} a_{5}^{27}+a_{5}^{163}+a_{5}^{67}+a_{5}^{51}+a_{2}^{2} a_{5}^{27}+a_{2}^{8} a_{5}^{3} \\
& =a_{5}^{67}+a_{5}^{51}+a_{5}^{36}=a_{5}^{35}\left(a_{5}^{32}+a_{5}^{16}+a_{5}\right) .
\end{aligned}
$$

and $0=\left(a_{5}^{32}+a_{5}^{16}+a_{5}\right)^{8}=a_{5}^{2}+a_{5}+a_{5}^{8}$. So $a_{5}$ is a root of the Conway polynomial $x^{7}+x+1$ in $\mathbb{F}_{128}$, and a generator of the multiplicative group $\mathbb{F}_{128}^{*}$. The following codes take an arbitrary root $e$ of $x^{7}+x+1$ as the inputting value of $a_{5}$.

$\mathrm{F} .\langle\mathrm{e}\rangle=\mathrm{GF}\left(2 \wedge 7\right.$, modulus $\left.=\mathrm{x}^{\wedge} 7+\mathrm{x}+1\right)$

L. $\langle$ a2 $\rangle=$ PolynomialRing $(F)$

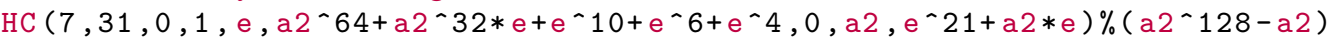

Here " $\%$ " is the modulus operator, giving the reduction of a polynomial (in $\mathbb{F}_{128}\left[a_{2}\right]$ ) modulo $a_{2}^{128}-a_{2}$. However, the output is a non-zero constant $e^{2}+e$ in $\mathbb{F}_{128}$. This makes a contradiction.

- Suppose $a_{3}=a_{5}^{3}$. Note that

$$
\begin{aligned}
0 & =\mathbf{H C}\left(7,23,0,1, a_{5}, a_{4}, a_{5}^{3}, a_{2}, a_{1}\right) \\
& =a_{5}^{15}+a_{5}^{11}+a_{4}^{2} a_{5}^{7}+a_{2}^{2} a_{5}^{3}+a_{1}^{2} a_{5} \\
& =a_{5}\left(a_{5}^{7}+a_{5}^{5}+a_{4} a_{5}^{3}+a_{2} a_{5}+a_{1}\right)^{2} .
\end{aligned}
$$

So $a_{1}=a_{5}^{7}+a_{5}^{5}+a_{4} a_{5}^{3}+a_{2} a_{5}$, and

$$
f(x)=x^{8}+x^{6}+a_{5} x^{5}+a_{4} x^{4}+a_{5}^{3} x^{3}+a_{2} x^{2}+\left(a_{5}^{7}+a_{5}^{5}+a_{4} a_{5}^{3}+a_{2} a_{5}\right) x .
$$


Note that $f\left(a_{5}\right)=0=f(0)$ with $a_{5} \neq 0$. So $f$ is not a PP over $\mathbb{F}_{128}$.

(3) Suppose $\left(a_{7}, a_{6}\right)=(1,0)$. Note that

$$
\begin{aligned}
& 0=\mathbf{H C}\left(7,19,1,0, a_{5}, a_{4}, a_{3}, a_{2}, a_{1}\right)=a_{5}^{3}+a_{4}^{2}+a_{1}, \\
& 0=\mathbf{H C}\left(7,37,1,0, a_{5}, a_{4}, a_{3}, a_{2}, a_{1}\right)=a_{3}^{33}+a_{2} .
\end{aligned}
$$

So $a_{1}=a_{5}^{3}+a_{4}^{2}$ and $a_{2}=a_{3}^{33}$. Also note that

$$
0=\mathbf{H C}\left(7,27,1,0, a_{5}, a_{4}, a_{3}, a_{3}^{33}, a_{5}^{3}+a_{4}^{2}\right)=a_{4}^{16} a_{5}^{8}+a_{3}^{8} a_{5}^{16}+a_{3}^{16}=\left(a_{4}^{2} a_{5}+a_{3} a_{5}^{2}+a_{3}^{2}\right)^{8} .
$$

So $a_{4}^{2} a_{5}+a_{3} a_{5}^{2}+a_{3}^{2}=0$. Thus $a_{4}=a_{3}^{64} a_{5}^{64}+a_{3} a_{5}^{63}$, and $a_{1}=a_{5}^{3}+a_{3} a_{5}+a_{3}^{2} a_{5}^{126}$. Then $\left(a_{3}, a_{5}\right) \in \mathbb{F}_{128} \times \mathbb{F}_{128}^{*}$ is a common solution of three equations:

$$
\left\{\begin{array}{l}
\mathbf{H C}\left(7,23,1,0, a_{5}, a_{3}^{64} a_{5}^{64}+a_{3} a_{5}^{63}, a_{3}, a_{3}^{33}, a_{5}^{3}+a_{3} a_{5}+a_{3}^{2} a_{5}^{126}\right)=0, \\
\mathbf{H C}\left(7,29,1,0, a_{5}, a_{3}^{64} a_{5}^{64}+a_{3} a_{5}^{63}, a_{3}, a_{3}^{33}, a_{5}^{3}+a_{3} a_{5}+a_{3}^{2} a_{5}^{126}\right)=0, \\
\mathbf{H C}\left(7,31,1,0, a_{5}, a_{3}^{64} a_{5}^{64}+a_{3} a_{5}^{63}, a_{3}, a_{3}^{33}, a_{5}^{3}+a_{3} a_{5}+a_{3}^{2} a_{5}^{126}\right)=0,
\end{array}\right.
$$

which can be solved by the following SageMath codes:

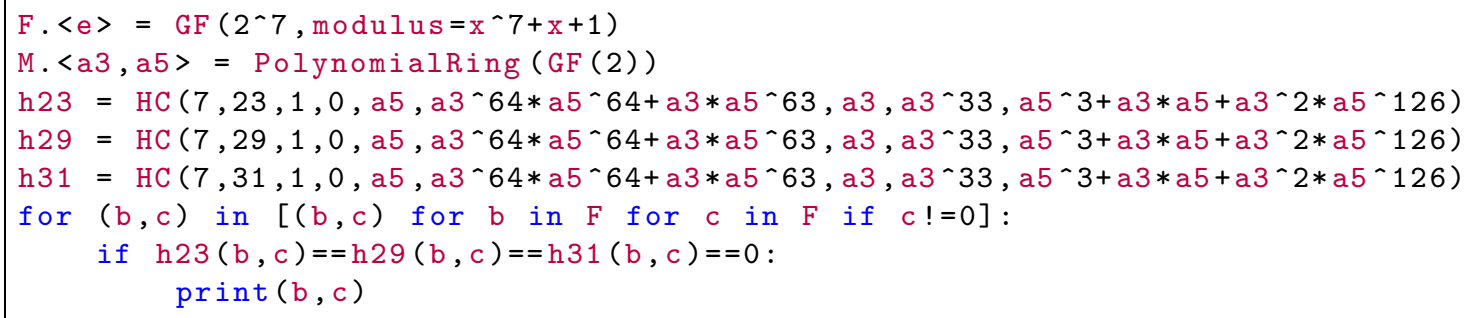

Nothing is printed in the output, which means that these three equations on $\left(a_{3}, a_{5}\right)$ have no common solution in $\mathbb{F}_{128} \times \mathbb{F}_{128}^{*}$. This makes a contradiction.

4.5. Case $r=8, q=256$.

Theorem 12. All PPs of degree 8 over $\mathbb{F}_{256}$ are exceptional.

Proof. Let us prove it by reduction to absurdity. Suppose that $f$ is a non-exceptional PP of degree 8 over $\mathbb{F}_{256}$. Without loss of generality, we can assume that $f(x)=x^{8}+\sum_{i=1}^{7} a_{i} x^{i}$ with all $a_{i} \in \mathbb{F}_{256}$ satisfying requirements (R1) of Proposition 3. As we mentioned in the introduction section, a linearized PP must be exceptional. In particular, we note that:

- $\left(a_{7}, a_{6}, a_{5}, a_{3}\right) \neq(0,0,0,0)$;

- $\left(a_{7}, a_{6}\right) \in\{(1,0),(0,1),(0,0)\}$.

Note that

$$
\begin{aligned}
& \mathbf{H C}\left(8,51,0,0, a_{5}, a_{4}, a_{3}, a_{2}, a_{1}\right)=a_{5}^{51}, \\
& \mathbf{H C}\left(8,55,0,0,0, a_{4}, a_{3}, a_{2}, a_{1}\right)=a_{3}^{37} .
\end{aligned}
$$

If $a_{7}=a_{6}=0$, then $a_{5}=a_{3}=0$. So $\left(a_{7}, a_{6}\right) \neq(0,0)$. Thus $\left(a_{7}, a_{6}\right)=(0,1)$ or $(1,0)$.

(1) Suppose $\left(a_{7}, a_{6}\right)=(0,1)$. Note that

$$
\begin{aligned}
& 0=\mathbf{H C}\left(8,43,0,1, a_{5}, a_{4}, a_{3}, a_{2}, a_{1}\right)=a_{5}^{3}+a_{3}, \\
& 0=\mathbf{H C}\left(8,85,0,1, a_{5}, a_{4}, a_{3}, a_{2}, a_{1}\right)=a_{3}^{85}+1 .
\end{aligned}
$$


So $a_{3}=a_{5}^{3} \neq 0$. Also note that

$$
\begin{aligned}
0 & =\mathbf{H C}\left(8,47,0,1, a_{5}, a_{4}, a_{5}^{3}, a_{2}, a_{1}\right)^{4} \cdot a_{5}^{3}+\mathbf{H C}\left(8,61,0,1, a_{5}, a_{4}, a_{5}^{3}, a_{2}, a_{1}\right) \\
& =a_{5}^{191}+a_{5}^{175}+a_{4}^{8} a_{5}^{159}+a_{2}^{8} a_{5}^{143}+a_{1}^{8} a_{5}^{135} \\
& =a_{5}^{135}\left(a_{5}^{7}+a_{5}^{5}+a_{4} a_{5}^{3}+a_{2} a_{5}+a_{1}\right)^{8} .
\end{aligned}
$$

So $a_{1}=a_{5}^{7}+a_{5}^{5}+a_{4} a_{5}^{3}+a_{2} a_{5}$, and

$$
f(x)=x^{8}+x^{6}+a_{5} x^{5}+a_{4} x^{4}+a_{5}^{3} x^{3}+a_{2} x^{2}+\left(a_{5}^{7}+a_{5}^{5}+a_{4} a_{5}^{3}+a_{2} a_{5}\right) x .
$$

Note that $f\left(a_{5}\right)=0=f(0)$ with $a_{5} \neq 0$. So $f$ is not a PP over $\mathbb{F}_{256}$.

(2) Suppose $\left(a_{7}, a_{6}\right)=(1,0)$. Then $\mathbf{H C}\left(8,37,1,0, a_{5}, a_{4}, a_{3}, a_{2}, a_{1}\right)=a_{3}=0$. Note that

$$
\begin{aligned}
0=\mathbf{H C}\left(8,53,1,0, a_{5}, a_{4}, 0, a_{2}, a_{1}\right) & =a_{2}^{4} a_{5}^{48}+a_{2}^{4} a_{4}^{32}+a_{1}^{16} a_{2}^{4} \\
& =a_{2}^{4}\left(a_{5}^{3}+a_{4}^{2}+a_{1}\right)^{16} .
\end{aligned}
$$

Therefore, either $a_{2}=0$ or $a_{1}=a_{5}^{3}+a_{4}^{2}$. Also note that

$$
\begin{aligned}
0=\mathbf{H C}\left(8,43,1,0, a_{5}, a_{4}, 0, a_{2}, a_{1}\right) & =a_{2}^{8} a_{5}^{3}+a_{2}^{8} a_{4}^{2}+a_{1} a_{2}^{8}+a_{1}^{8} \\
& =a_{2}^{8}\left(a_{5}^{3}+a_{4}^{2}+a_{1}\right)+a_{1}^{8}=a_{1}^{8} .
\end{aligned}
$$

So $a_{1}=0$. Then

$$
0=\mathbf{H C}\left(8,45,1,0, a_{5}, a_{4}, 0, a_{2}, 0\right)=a_{5}^{32}+a_{2}^{12},
$$

and thus $a_{5}=\left(a_{5}^{32}\right)^{8}=a_{2}^{96}$. Further, if $a_{2}=0$, then $a_{5}=a_{2}^{96}=0$, and $a_{4}=0$ as

$$
\mathbf{H C}\left(8,39,1,0,0, a_{4}, 0,0,0\right)=a_{4}^{6},
$$

while $f(x)=x^{8}+x^{7}$ is not a PP. Therefore, $a_{2} \neq 0$ in this case. So $a_{4}^{2}=a_{5}^{3}=\left(a_{2}^{96}\right)^{3}=a_{2}^{288}$, and $a_{4}=a_{2}^{144}$. As

$$
0=\mathbf{H C}\left(8,39,1,0, a_{2}^{96}, a_{2}^{144}, 0, a_{2}, 0\right)=a_{2}^{386}+a_{2}^{4} .
$$

we have $a_{2}^{382}=1$, and $a_{2}=1$ as $\operatorname{gcd}(382,255)=1$. Then $a_{5}=a_{4}=a_{2}=1, a_{3}=a_{1}=0$, and

$$
\mathbf{H C}(8,55,1,0,1,1,0,1,0)=1 \neq 0 \text { in } \mathbb{F}_{256} \text {. }
$$

This makes a contradiction.

4.6. Case $r=9, q=512$.

Theorem 13. All PPs of degree 8 over $\mathbb{F}_{512}$ are exceptional.

Proof. Let us prove it by reduction to absurdity. Suppose that $f$ is a non-exceptional PP of degree 8 over $\mathbb{F}_{512}$. Without loss of generality, we can assume that $f(x)=x^{8}+\sum_{i=1}^{7} a_{i} x^{i}$ with all $a_{i} \in \mathbb{F}_{512}$ satisfying requirements $(\mathbf{R 1}),(\mathbf{R 2})$ and (R3) of Proposition 3. As we mentioned in the introduction section, a linearized PP must be exceptional. In particular, we note that:

- $\left(a_{7}, a_{6}, a_{5}, a_{3}\right) \neq(0,0,0,0)$;

- $\left(a_{7}, a_{6}\right) \in\{(1,0),(0,1),(0,0)\}$;

- if $\left(a_{7}, a_{6}, a_{5}\right)=(0,1,0)$, then $a_{4}=0$;

- if $a_{7}=a_{6}=0 \neq a_{5}$, then $\left(a_{5}, a_{4}\right)=(1,0)$. 
As $\mathbf{H C}\left(9,73, a_{7}, a_{6}, a_{5}, a_{4}, a_{3}, a_{2}, a_{1}\right)=a_{7}^{73}=0,\left(a_{7}, a_{6}\right)=(0,0)$ or $(0,1)$. Note that

$$
\mathbf{H C}\left(9,117,0,0,0, a_{4}, a_{3}, a_{2}, a_{1}\right)=a_{3}^{85} .
$$

If $a_{6}=a_{5}=0$, then $a_{3}=0$. Recall that $\left(a_{7}, a_{6}, a_{5}, a_{3}\right) \neq(0,0,0,0)$, so $\left(a_{6}, a_{5}\right) \neq(0,0)$.

(1) Suppose $a_{7}=a_{6}=0 \neq a_{5}$. Then $\left(a_{5}, a_{4}\right)=(1,0)$. Note that

$$
\begin{aligned}
& \mathbf{H C}\left(9,93,0,0,1,0, a_{3}, a_{2}, a_{1}\right)=a_{3}, \\
& \mathbf{H C}\left(9,103,0,0,1,0,0, a_{2}, a_{1}\right)=a_{1}, \\
& \mathbf{H C}\left(9,107,0,0,1,0,0, a_{2}, 0\right)=a_{2}^{8} .
\end{aligned}
$$

So $a_{3}=a_{1}=a_{2}=0$, and $f(x)=x^{8}+x^{5}$ is not a PP over $\mathbb{F}_{512}$.

(2) Suppose $\left(a_{7}, a_{6}, a_{5}\right)=(0,1,0)$. Then $a_{4}=0$. Note that

$$
\begin{aligned}
& \mathbf{H C}\left(9,171,0,1,0,0,0, a_{2}, a_{1}\right)=a_{2}, \\
& \mathbf{H C}\left(9,183,0,1,0,0,0,0, a_{1}\right)=a_{1}^{16} .
\end{aligned}
$$

If $a_{3}=0$, then $a_{2}=a_{1}=0$ and $f(x)=x^{8}+x^{6}$ is not a PP. So $a_{3} \neq 0$. Note that

$$
\begin{aligned}
0 & =\mathbf{H C}\left(9,87,0,1,0,0, a_{3}, a_{2}, a_{1}\right)=a_{3}^{5}+a_{2}^{2} a_{3}+a_{1} a_{3}^{2}, \\
0 & =\mathbf{H C}\left(9,91,0,1,0,0, a_{3}, a_{2}, a_{1}\right)=a_{3}^{17}+a_{2}^{2} a_{3}^{9}+a_{1} a_{3}^{10}+a_{2}^{8} a_{3}, \\
0 & =\mathbf{H C}\left(9,87,0,1,0,0, a_{3}, a_{2}, a_{1}\right) \cdot a_{3}^{8}+\mathbf{H C}\left(9,91,0,1,0,0, a_{3}, a_{2}, a_{1}\right) \\
& =a_{3}^{17}+a_{3}^{13}+a_{2}^{8} a_{3}=a_{3}\left(a_{3}^{4}+a_{3}^{3}+a_{2}^{2}\right)^{4} .
\end{aligned}
$$

Let $a_{3}=t^{2}$ with $t \in \mathbb{F}_{512}^{*}$. Then $a_{2}=t^{4}+t^{3}, a_{1}=a_{3}^{3}+a_{2}^{2} a_{3}^{-1}=t^{4}$, and

$$
f(x)=x^{8}+x^{6}+t^{2} x^{3}+\left(t^{4}+t^{3}\right) x^{2}+t^{4} x .
$$

Note that $f(t+1)=t^{8}+t^{5}=f(t)$. So $f$ is not a PP over $\mathbb{F}_{512}$.

(3) Suppose $\left(a_{7}, a_{6}\right)=(0,1)$ and $a_{5} \neq 0$. Note that

$$
\begin{aligned}
0 & =\mathbf{H C}\left(9,87,0,1, a_{5}, a_{4}, a_{3}, a_{2}, a_{1}\right) \cdot\left(a_{5}^{96}+a_{3}^{32}\right)+\mathbf{H C}\left(9,103,0,1, a_{5}, a_{4}, a_{3}, a_{2}, a_{1}\right) \\
& =a_{5}^{115}+a_{3} a_{5}^{112}+a_{3}^{32} a_{5}^{19}+a_{3}^{33} a_{5}^{16}=a_{5}^{16}\left(a_{5}^{3}+a_{3}\right)^{33} .
\end{aligned}
$$

So $a_{3}=a_{5}^{3}$. Also note that

$$
\begin{aligned}
0 & =\mathbf{H C}\left(9,87,0,1, a_{5}, a_{4}, a_{5}^{3}, a_{2}, a_{1}\right) \\
& =a_{5}^{15}+a_{5}^{11}+a_{4}^{2} a_{5}^{7}+a_{2}^{2} a_{5}^{3}+a_{1}^{2} a_{5} \\
& =a_{5}\left(a_{5}^{7}+a_{5}^{5}+a_{4} a_{5}^{3}+a_{2} a_{5}+a_{1}\right)^{2} .
\end{aligned}
$$

So $a_{1}=a_{5}^{7}+a_{5}^{5}+a_{4} a_{5}^{3}+a_{2} a_{5}$, and

$$
f(x)=x^{8}+x^{6}+a_{5} x^{5}+a_{4} x^{4}+a_{5}^{3} x^{3}+a_{2} x^{2}+\left(a_{5}^{7}+a_{5}^{5}+a_{4} a_{5}^{3}+a_{2} a_{5}\right) x .
$$

Note that $f\left(a_{5}\right)=0=f(0)$ with $a_{5} \neq 0$. So $f$ is not a PP over $\mathbb{F}_{512}$.

Acknowledgements. This work was supported by the Natural Science Foundation of Guangdong Province [No. 2018A030310080]. The author was also sponsored by the National Natural Science Foundation of China [No. 11801579]. Special thanks go to my lovely newborn daughter, without whose birth should this paper have come out much earlier. 


\section{REFERENCES}

[1] Bartoli, D., Giulietti, M., Quoos, L., \& Zini, G. (2017). Complete permutation polynomials from exceptional polynomials. J. Number Theory, 176, 46-66. doi:10.1016/j.jnt.2016.12.016.

[2] Chahal, J. S., \& Ghorpade, S. R. (2018). Carlitz-Wan conjecture for permutation polynomials and Weill bound for curves over finite fields. Finite Fields Appl., 54, 366-375. doi:10.1016/j.ffa.2018.07.006.

[3] Davenport, H., \& Lewis, D. J. (1963). Notes on congruences. I. Quart. J. Math. Oxford Ser. (2), 14, 51-60. doi:10.1093/qmath/14.1.51.

[4] Dickson, L. E. (1896/97). The analytic representation of substitutions on a power of a prime number of letters with a discussion of the linear group. Ann. of Math., 11, 65-120. doi:10.2307/1967217.

[5] Fan, X. (2018). A classification of permutation polynomials of degree 7 over finite fields. arXiv: 1812.02080.

[6] Fan, X. (2018). The Weil bound and non-exceptional permutation polynomials over finite fields. arXiv:1811.12631.

[7] von zur Gathen, J. (1991). Values of polynomials over finite fields. Bull. Austral. Math. Soc., 43, 141-146. doi:10.1017/S0004972700028860.

[8] Hayes, D. R. (1967). A geometric approach to permutation polynomials over a finite field. Duke Math. J., 34, 293-305. doi:10.1215/S0012-7094-67-03433-3.

[9] Hermite, C. (1863). Sur les fonctions de sept lettres. C. R. Acad. Sci. Paris, 57, 750-757.

[10] Li, J., Chandler, D. B., \& Xiang, Q. (2010). Permutation polynomials of degree 6 or 7 over finite fields of characteristic 2. Finite Fields Appl., 16, 406-419. doi:10.1016/j.ffa.2010.07.001.

[11] Lidl, R., \& Niederreiter, H. (1997). Finite fields volume 20 of Encyclopedia of Mathematics and its Applications. (2nd ed.). Cambridge University Press, Cambridge. doi:10.1017/CB09780511525926 with a foreword by P. M. Cohn.

[12] Lucas, E. (1878). Theorie des Fonctions Numeriques Simplement Periodiques. [Continued]. Amer. J. Math., 1, 197-240. doi:10.2307/2369311.

[13] The Sage Developers (2017). SageMath, the Sage Mathematics Software System (Version 7.6). URL: http://www. sagemath.org. doi:10.5281/zenodo. 593563.

[14] Wan, D. Q. (1987). On a conjecture of Carlitz. J. Austral. Math. Soc. Ser. A, 43, 375-384. doi:10.1017/S1446788700029657.

[15] Wan, D. Q. (1993). A p-adic lifting lemma and its applications to permutation polynomials. In Finite fields, coding theory, and advances in communications and computing (Las Vegas, NV, 1991) (pp. 209-216). Dekker, New York volume 141 of Lecture Notes in Pure and Appl. Math.. URL: http://www.math.uci.edu/ dwan/lift.pdf. 